\title{
Primary Central Nervous System T-Cell Lymphoma of the Brain
}

\author{
Mandana Behbahani and Mark K. Lyons*
}

5777 East Mayo Boulevard. Mayo Clinic Hospital 5-East Phoenix AZ 85054 USA

\begin{abstract}
Primary central nervous system (PCNSL) T-cell lymphoma is a very rare extra nodal non-Hodgkin's lymphoma (NHL) tumor arising from the central nervous system. The incidence of this disease is increasing globally in part due to larger numbers of immunocompromised patients. There are no pathognomonic clinical or radiographic features of T-cell PCNSL. Brain biopsy is generally necessary for tissue diagnosis in order to direct appropriate treatment and counsel patients on prognosis. We report our experience in an immunocompetent patient who presented with a several week history of progressive encephalopathy. The imaging findings, histopathological studies, treatment and review of the current literature of this rare condition are discussed.
\end{abstract}

Keywords: Brain biopsy, CNS lymphoma, T-cell.

\section{INTRODUCTION}

Primary central nervous system lymphoma (PCNSL) refers to an extra nodal non-Hodgkin's lymphoma (NHL) tumor that primarily arises from the central nervous system (CNS) without evidence of systemic disease. Primary central nervous system T-cell lymphoma is considered a distinct disease [1]. It remains a very rare entity despite a gradual increase in global incidence. The reason for the initial rise in incidence had been linked to a growing immunocompromised population, in part due to, acquired immune deficiency syndrome (AIDS), increased prevalence of human immunodeficiency virus (HIV) infection, organ transplantation and the elderly [2-4]. However, due to advances in antiretroviral therapy the incidence of PCNSL in the immunocompromised population has been decreasing, yet the incidence of PCNSL in the immunocompetent population has been rising without clear explanation. The overall occurrence rate of PCNSL is 1:100,000, which accounts for 3-6\% of all primary brain neoplasms $[2,5]$. The occurrence rate of B-cell lymphomas is much more common than T-cell lymphomas and this concept hold true both in systemic and CNS presentation of lymphomas $[2,5,6]$. Of the cases of PCNSL reported, $98 \%$ are B-cell and 2\% are T-cell lymphomas [3, 6 ]. The prevalence of T-cell PCNSL is much higher in eastern countries, such as Korea (16\%) and Japan (8-14\%) compared to that of western countries such as France $(3.6 \%)$ and the United States. (2\%) [5].

There have been recent efforts to classify T-cell and Bcell PCNSL based upon presentation, radiological findings and epidemiological factors. Some have reported that factors such as age, sex, location of the tumor and presenting symptoms may significantly impact clinical outcome [5]. A recent radiological overview on T-cell PCNSL by Kim and coworkers has shown predilection for sub-cortical location, high incidence of cortical or intra-tumoral hemorrhage, rim

*Address correspondence to this author at the 5777 East Mayo Boulevard. Mayo Clinic Hospital 5-East Phoenix AZ 85054 USA; Tel: 480.342.3616; Fax: 480.342.3699; E-mail: lyons.mark2@mayo.edu enhancement or cystic area consistent with necrosis on MRI [7]. In addition, they identified lower relative cerebral blood flow (rCBV) as a distinguishing marker between both B-cell and T-cell PCNSL versus other brain tumors, such as high grade glioma [7]. However, these diagnostic findings are neither sensitive nor specific enough and therefore diagnosis usually depends upon obtaining tissue sample through biopsy [8]. There remains disagreement in the literature regarding the prognosis of T-cell PCNSL and whether it is more favorable, less favorable or equivalent prognosis relative to other primary CNS lymphomas $[4,5,9,10]$. We report a case of primary T-cell lymphoma of the central nervous system involving the brain with a review of the literature in this field. The resulting literature does not yet provide unequivocal answers but opens new investigatory avenues concerning pathophysiology, imaging modalities, diagnostic and therapeutic responses.

\section{CASE REPORT}

We report the case of a 67 year old left handed female who presented to our institution with a two week history of progressive confusion and expressive dysphasia. The patient began having difficulty with directions and getting lost while driving in familiar areas. Her past medial history was significant for type 2 diabetes mellitus, hypertension and hypothyroidism. Her neurologic examination was notable for mild confusion scoring 34/38 on the Kokem mental status test, word-finding difficulty and encephalopathy. Laboratory studies were normal and computerized tomography scan of the brain was unremarkable. An electroencephalogram revealed generalized slowing with prominence in the right centrotemporal region. There were no epileptiform discharges. A magnetic resonance (MR) imaging study of the brain with gadolinium demonstrated extensive cortical and subcortical lesions involving the supratentorial and infratentorial compartments (Figs. 1A-B). Extensive associated contrast enhancement of the lesions was present with minimal diffusion restriction. A MR angiogram was normal. Cerebrospinal fluid analysis was notable for 20 red blood cells; glucose $85 \mathrm{mg} / \mathrm{dl}$; protein $48 \mathrm{mg} / \mathrm{dl}$ (normal range $14-45 \mathrm{mg} / \mathrm{dl}$ ); 20 white blood cells (normal range $<5$ ) and negative cytology. 


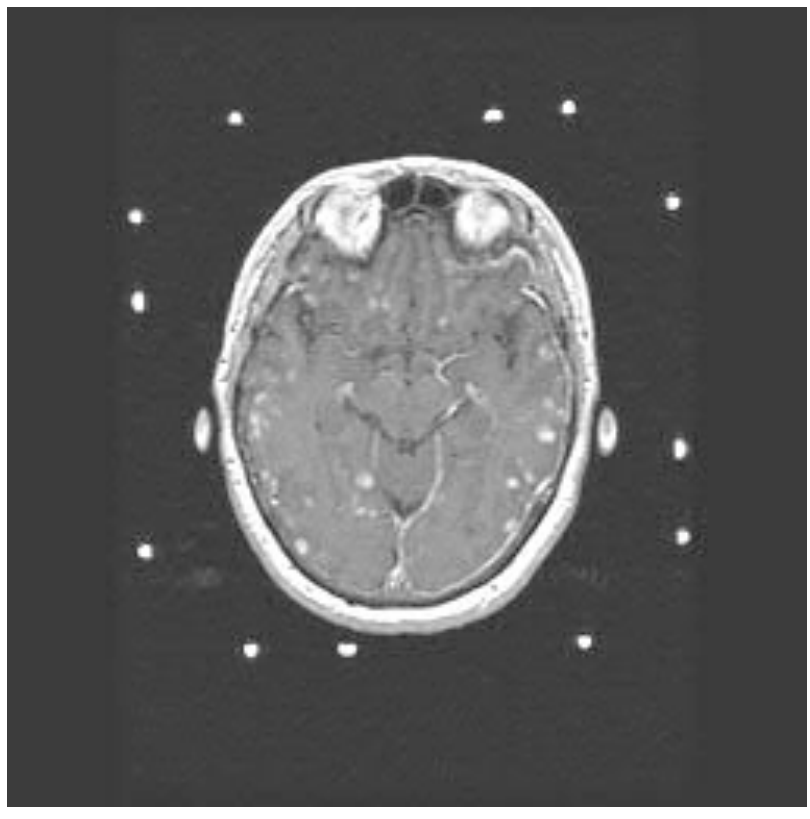

A

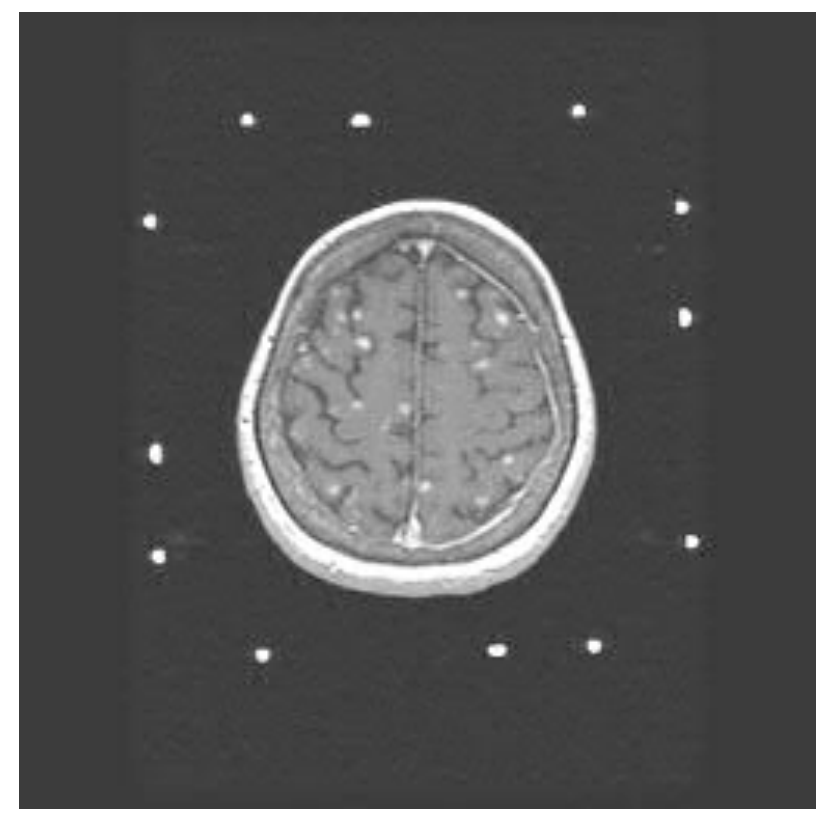

B

Fig (1). A-B: T-1 weighted axial gadolinium enhanced MR images demonstrating multiple small enhancing cortical and subcortical lesions.

The patient underwent a stereotactic brain biopsy of one of the right frontal lesions. The pathology was consistent with T-cell lymphoma. Immunohistochemical stains were performed on paraffin-embedded tissue using antibodies to CD2, CD3, CD5, CD7, CD8, CD20, CD30, CD31, CD43, CD79a, KP-1 (CD68), S-100, PAX5, TIA-1, ALK, EMA, TcR beta $\mathrm{F} 1$ and TcR gamma/delta. Tumor cells reacted positively to CD2, CD3, CD5, CD7, CD8, CD43 and TIA-1. The remaining stains were negative. There was a high proliferation index by Ki67 staining ( $>60 \%)$. Additional imaging included enhanced CT scans of the chest, abdomen and pelvis as well as positron emission tomography were negative. Bone marrow aspirates were negative. Following discussion of the chemotherapy and radiotherapy treatment options with the patient and family, they opted for a chemotherapy regimen of cyclophosphamide, hyroxydaunorubicin, vincristine and prednisone (CHOP) and is currently in remission at eight months following her initial diagnosis. She did not receive radiation therapy.

\section{DISCUSSION}

Presentation of T-cell PCNSL is variable between patients suffering from AIDS and other immunocompromised patients and immunocompetent population. The most common presenting symptoms of T-cell PCNSL are: motor weakness and incoordination, disturbance of consciousness, headache, nausea, visual disturbances, seizures, aphasia, sensory symptoms, autonomic dysfunction, hypothalamic dysfunction and ataxia [11]. A slight male preponderance has been reported with male to female ratio of 1.5:1 [12]. The order of radiographic location of T-cell PCNSL tumors presentation in decreasing frequency is: supratentorial, infratentorial, basal ganglia nuclei, brainstem and leptomeninges $[11,13]$.

Unlike most immunocompetent patients, AIDS patients have a predilection for infra-tentorial location of tumors
[14]. Immunocompromised patients will more often present with multiple lesions and immunocompetent patients will more often harbor a single lesion. However, as in our patient who had multiple lesions despite being immunocompetent, it highlights the fact that PCNSL can present in many different forms. Cystic degeneration with necrosis and hemorrhage are consistent within both populations [3]. T-cell PCNSL has consistent histological features including small nuclei, bland morphology without high grade features, heterogeneity and angiocentricity $[2,3,5,10-12]$. The histopathologic appearance of T-cell PCNSL can include loosely scattered cells without solid mass formation and frequent perivascular infiltration [2]. Elevated lactate dehydrogenase and CSF protein levels have been noted to potentially be poor prognostic indicators [5]. Overall, however, there has been no definite pattern of T-cell PCNSL emergence clinically, radiologically or pathologically [7].

The treatment of patients with corticosteroids prior to brain biopsy has been shown in some cases to induce apoptosis of lymphoid cell and deplete brain tissue of neoplastic infiltrate within 24-48 hours of initiation of the medication, resulting in only prominent gliosis with only a few reactive lymphocytes or macrophages in the brain biopsy specimen $[14,15]$. Several different tests including CD7 surface antigen loss, immuno-staining for CD3, CD4, CD8, CD20 and molecular testing for T-cell receptor (TCR) gene rearrangements of both TCR- $\gamma$ and $\beta$ are employed in order to diagnose T-cell PCNSL [3]. These methods are especially useful in distinguishing between neoplasia and a reactive process such as demyelination and vasculitis [6]. Monoclonal lymphoid cell proliferation is often found in cases of T-cell PCNSL [6]. There have been four case reports of Ki-1 antigen expression in anaplastic large cell lymphomas that have shown poor prognosis and resistance to corticosteroids [15].

Most PCNSL are of B-cell origin with T-cell PCNSL representing less than 5\% of PCNSL [16-20]. As such, the 
optimal treatment regimen for T-cell PCNSL is evolving. Previous studies analyzing the prognostic factors and treatment paradigms for patients with aggressive systemic T-cell lymphoma have reported that CHOP chemotherapy appears to be as effective, and with potentially less side effects, than more intensive therapies [18, 19]. The excellent study by Shenkier and colleagues summarized the largest series of global reports of PCNSL of T-cell origin to date [17]. They identified 45 patients concluding that the presentation and outcome appeared to be similar to B-cell PCNSL and that performance status and administration of methotrexate were associated with better outcomes [17]. However, although methotrexate was the most commonly administered medication, the individual treatment regimens were very different. While 29 of the 45 of the patients (64\%) received methotrexate, 18 of the 29 patients $(62 \%)$ in that group also received other various chemotherapy agents. The methotrexate was delivered intravenously, intrarteriorly or intrathecally. Thirty-five of the 45 patients (78\%) also underwent radiation therapy. The mean disease specific survival was 25 months [17]. Consequently, the treatment paradigms varied considerably across the participating institutions. Levin and co-workers subsequently published their series of five patients with T-cell PCNSL who presented with leptomeningeal spread and neurolymphomatosis [20]. Four of the five patients received high dose methotrexate, procarbazine and lomustine combined with intrathecal cytarabine. The other patient received cyclophosphamide/ doxorubicin and intrathecal methotrexate. All patients underwent salvage therapy with either whole brain radiation or additional chemotherapy. Mean survival was 16 months for the four patients who died and one was alive at 36 months. It appears that treatment with methotrexate may be associated with better survival in T-cell PCNSL, but the paucity of cases and variety of treatment regimens requires further study.

The exact pathophysiology of both B-cell and T-cell PCNSL remains unknown. It has been postulated that since PCNSL generally arises from the superficial sub-cortical area (as seen in our patient), it raises the possibility that pathogenesis of PCNSL differs from that of diffuse large cell lymphoma [12]. Other literature suggests that the vascular and peri-vascular microenvironment in PCNSL may be responsible either B-cell or T-cell recruitment due to upregulation of a chemo-attractant the either recruits cells from the periphery or traps existing lymphocytes within the CNS [21]. Treatment of this neoplasm has improved disease control in PCNSL achieving survival of 40-60 months on average [8, 9, $13,22]$. Radiation and corticosteroids have been reported to achieve complete response in $20-50 \%$ of patient with median survival of 13.5 months [4]. Chemotherapy has shown the best outcomes over time relative to other therapeutic approaches [11]. The combined method of chemotherapy and radiation is also used in rare cases of primary peripheral nervous system lymphoma with primary CNS metastasis [15].

\section{CONCLUSION}

The prognosis of T-cell PCNSL has been controversial without a consensus in the literature. Some reports indicate poor prognosis relative to other CNS lymphomas and others reports better or comparable overall survival time $[8,10,13]$.
Our immunocompetent patient presented with a progressive course of cognitive decline with encephalopathic features. Magnetic resonance imaging of the brain revealed small multiple enhancing subcortical lesions. She has responded well to chemotherapy. This report highlights the rarity of this condition and the review of the literature illustrates the variety of ways in which this condition may present. Brain biopsy was necessary for diagnosis and led to the appropriate treatment.

\section{ACKNOWLEDGEMENT OF FUNDING}

None declared.

\section{ANY CONFLICTS OF INTEREST}

None declared.

\section{REFERENCES}

[1] Swedlow SH, Campo E, Harris NL, et al. World Health Organization Classification of tumours. Pathology and genetics of tumours of haematopoietic and lymphoid tissues. WHO press: WHO classification of tumours of haematopoietic and lymphoid tissues. $4^{\text {th }}$ ed. WHO Press, Lyon, France 2008; pp. 236-237.

[2] Choi JS, Nam DH, Ko YH, et al. Primary central nervous system lymphoma in Korea: comparison of B- and T-cell lymphomas. Am J Surg Pathol 2003; 27: 919-28.

[3] Dulai MS, Park CY, Howell WD, et al. CNS T-cell lymphoma: an under-recognized entity? Acta Neuropathol 2008; 115: 345-56.

[4] Latta S, Myint ZW, Jallad B. Primary central nervous system T-cell lymphoma in AIDS patients: case report and literature review. Curr Oncol 2010; 17(5): 63-6.

[5] Shenkier TN, Blay JY, O'Neill BP, et al. Primary CNS lymphoma of T-cell origin: a descriptive analysis from the international primary CNS lymphoma collaborative group. J Clin Oncol 2005; 23(10): 2233-339.

[6] Harder A, Dudel C, Anagnostopoulos I, et al. Molecular genetic diagnosis of a primary central nervous system $\mathrm{T}$ cell lymphoma. Acta Neuropathol 2003; 105: 65-8.

[7] Kim EY, Kim SS. Magnetic resonance findings of primary central nervous system T-cell lymphoma in immunocompetent patients. Acta Radiol 2005; 46: 187-92.

[8] Soussain C, Hoang-Xuan K. Primary central nervous system lymphoma: an update. Curr Opin Oncol 2009; 6: 550-8.

[9] Gijtenbeek JM, Rosenblum MK, DeAngelis LM. Primary central nervous system T-cell lymphoma. Neurology 2001; 57: 716-8.

[10] Mineura K, Sawataishi J, Sasajima T, et al. Primary central nervous system involvement of the so called "peripheral T-cell lymphoma": report of a case and review of the literature. J Neurooncol 1993; 16: 235-42.

[11] Pulsoni A, Gubitosi G, Rocchi L, et al. Primary T-cell lymphoma of central nervous system (PTCLCNS): a case with unusual presentation and review of the literature. Ann Oncol 1999; 10(12): 151923.

[12] Da Silva AN, Lopes MB, Schiff D. Rare pathological variants and presentations of primary central nervous system lymphomas. Neurosurg Focus 2006; 21: 1-6.

[13] Ponce J, Segura A, Gimenez A, et al. Primary meningeal lymphoma of T-cell origin: a rare presentation of primary central nervous system lymphomas. Clin Lymphoma Myeloma 2007; 7(8): 546-9.

[14] Bindal AK, Blisard KS, Melin-Aldama H, Warnick RE. Primary Tcell lymphoma of the brain in acquired immunodeficiency syndrome: case report. J Neurooncol 1997; 31: 267-71.

[15] Goldbrunner R, Warmuth-Metz M, Tonn JC, et al. Primary Ki-1positive T-cell lymphoma of the brain-an aggressive subtype of lymphoma: case report and review of the literature. Surg Neurol 1996; 46: 37-41.

[16] Gijtenbeek JMM, Rosenblum MK, DeAngelis LM. Primary central nervous system T-cell lymphoma. Neurology 2001; 57: 716-8.

[17] Shenkier TN, Blay JY, O’Neill BP, et al. Primary CNS lymphoma of T-cell origin: a descriptive analysis from the international primary CNS lymphoma collaborative group. J Clin Oncol 2005; 23(10): 2233-9. 
[18] Pfreundschuh M, Trumper L, Kloess M, et al. Two-weekly or 3weekly CHOP chemotherapy with or without etoposide for the treatment of elderly patients with aggressive lymphomas: results of the NHL-B2 trial of the DSHNHL. Blood 2004; 104(3): 634-41.

[19] Escalon MP, Liu NS, Yang Y, et al. Prognostic factors and treatment of patients with T-cell non-Hodgkin lymphoma. Cancer 2005; 103(10): 2091-8.

[20] Levin N, Soffer D, Grissaru S, et al. Primary T-cell CNS lymphoma presenting with leptomeningeal spread and neurolymphomatosis. J Neurooncol 2008; 90: 77-83.
[21] Venetz D, Ponzoni M, Schiraldi M, et al. Perivascular expression of CXCL9 and CXCL12 in primary central nervous system lymphoma: T-cell infiltration and positioning of malignant B cells. Int J Cancer 2010; 127: 2300-12.

[22] Terasaki M, Abe T, Tajima Y, et al. Primary choroid plexus T-cell lymphoma and multiple aneurysms in the CNS. Leuk Lymphoma 2006; 47: 1680-2.

(C) Behbahani and Lyons; Licensee Bentham Open.

This is an open access article licensed under the terms of the Creative Commons Attribution Non-Commercial License (http://creativecommons.org/licenses/by-nc/3.0/) which permits unrestricted, non-commercial use, distribution and reproduction in any medium, provided the work is properly cited. 